\title{
A retrospective analysis of dysglycaemia and its risk factors in a cohort of human immunodeficiency virus infected antiretroviral therapy naïve children in Makurdi, Nigeria
}

\author{
Emmanuel Anigilaje ${ }^{1}$ \\ Sri Lanka Journal of Child Health, 2017; 46(3): 248-258
}

\begin{abstract}
Background: Human immunodeficiency virus (HIV) endocrinopathy involving the pancreas manifests clinically as dysglycaemia, including hypoglycaemia and hyperglycaemia. Dysglycaemia increases mortality in sick children, underlying the need for its evaluation and management.
\end{abstract}

Objective: To assess the prevalence and risk factors of dysglycaemia in a cohort of antiretroviral therapy (ART)-naïve children at the point of enrolment into a paediatric ART clinic of the Federal Medical Centre, Makurdi, Nigeria.

Method: A retrospective cross-sectional study was carried out between June 2010 and June 2012. Hypoglycaemia was defined as random blood glucose level $<2.2 \mathrm{mmol} / 1$ and hyperglycaemia as random blood glucose $>6.6 \mathrm{mmol} / 1$. Potential risk factors of dysglycaemia were tested for significance in bivariate and multivariate regression analyses. $P$ value less than 0.05 was considered to be significant.

Results: 429 children, aged 1-15 years, including 223 males and 206 females were studied. The median age was 5 years. Twelve $(2.8 \%)$ children had hypoglycaemia and 35 (8.2\%) had hyperglycaemia. In multivariate regression analysis, no factor significantly predicted the risk of hypoglycaemia, whereas children co-infected with hepatitis $\mathrm{C}$ were at a significant risk of hyperglycaemia (adjusted odds ratio; $2.06,95 \% \mathrm{CI}$; $1.05-8.52, \mathrm{P}=0.03$ ).

Conclusions: In this study HIV-infected Nigerian children who were not on ART had a low prevalence of hypoglycaemia but a high prevalence of hyperglycaemia. Hepatitis C co-infection was a significant independent risk factor for hyperglycaemia.

${ }^{1}$ Department of Paediatrics, College of Health Sciences, University of Abuja, Nigeria

Correspondence: demolaanigilaje@yahoo.co.uk

(Received on 24 December 2016: Accepted after revision on 17 February 2017)

The author declares that there are no conflicts of interest

Personal funding was used for the project.

Open Access Article published under the Creative

Commons Attribution CC-BY (cc) (P)
DOI: http://dx.doi.org/10.4038/sljch.v46i3.8327

(Key words: Dysglycaemia, risk factors, ART-naïve children, Nigeria)

\section{Introduction}

About 400,000 Nigerian children were living with human immunodeficiency virus (HIV) in $2013^{1}$. Before the access to Highly Active Anti-Retroviral Therapy (HAART), 50\% of perinatally HIV infected children died before their second birthday ${ }^{2}$. However, with the easy access to, and the success of HAART in reducing mortality from acquired immune deficiency syndrome (AIDS) $)^{3,4}$, the attendant increased longevity is making comorbidities like HIV-endocrinopathies more frequent ${ }^{5}$.

HIV-endocrinopathies involve the pituitary, the thyroid, the adrenal, the gonads and the pancreas ${ }^{6}$. HIV pancreatic dysfunctions manifest clinically as hypoglycaemia and hyperglycemia ${ }^{7-15}$ and are seen in both antiretroviral therapy (ART)-experienced and ART-naïve patients ${ }^{11,12}$. The mechanisms of HIV pancreatic dysfunctions include pancreatic infection by HIV and other opportunistic organisms including cytomegalovirus and toxoplasma ${ }^{7}$, infiltration of the pancreatic gland by malignancies ${ }^{7}$, and the effects of therapeutic interventions ${ }^{8-10}$. For example, pancreatic beta cell toxicity by pentamidine has been found to result in hypoglycaemia with hyperglycaemia and diabetes developing in the long term ${ }^{8}$. Also, reports of insulin resistance and the development of hyperglycaemia and overt diabetes increased with the routine clinical use of protease inhibitors $(\mathrm{PI})^{9}$ with a reversal of hyperglycaemia following the replacement of the PI with another class of medication ${ }^{10}$. Furthermore, evidence is also available suggesting the possibility of insulin resistance, as a HIV disease-associated component, among ART- naïve patients with AIDS $^{11}$. On the other hand, Hommes et al. ${ }^{12}$ reported that clinically stable HIV infected patients are protected against hyperglycaemia and diabetes as they were found to have increased sensitivity of peripheral tissues to insulin and increased nonoxidative glucose disposal ${ }^{12}$.

There is a dearth of data on dysglycaemia among HIV-infected children, although a Thailand study revealed that about $4 \%$ had hyperglycaemia in a 
cohort of ART experienced children (non-PI based $)^{13}$. Several studies ${ }^{14-17}$ among HIV-infected Nigerian children have reported a high prevalence of malnutrition, diarrhoeal disease, pneumonia, septicaemia and malaria, all of which are known confounders of hypoglycaemia ${ }^{18-22}$ and hyperglycaemia ${ }^{18,23,24}$ outside the context of HIV infection. Regardless of the aetiopathogenesis of hypoglycaemia and hyperglycaemia in HIVinfection, both have been associated with increased morbidity and mortality in severely ill and nonseverely ill children, underlining the need for their evaluation, whenever they may be present ${ }^{25,26}$.

\section{Objectives}

To retrospectively determine the prevalence and the associated risk factors of dysglycaemia (hypoglycaemia and hyperglycaemia) among ART naïve HIV infected children, at the point of recruitment into an ART programme, at the Federal Medical Centre (FMC), Makurdi, Nigeria, between June 2010 and June 2012.

\section{Method}

Ethics: Approval to use the data for this study was obtained from the Research and Ethics Committee of the FMC, Makurdi and the AIDS Prevention Initiative in Nigeria (APIN)/Harvard PEPFAR (The USA President's Emergency Plan for AIDS Relief) programme. The parents/legal care providers had earlier given written informed consent for the use of the childrens' data for research purposes at the recruitment of these children into the ART programme of the FMC, Makurdi. The process of obtaining the written informed consent was in accordance with the Helsinki Declaration of 1975 , as revised in 2000 .

Selection and study population: A minimum sample size of 384 was calculated using the Leslie Kish's $\operatorname{method}^{27}$ at a standard normal deviate of 1.96 , assuming 50\% target population and tolerating 5\% sampling error. A total of 429 children whose data were relevant to this study were all abstracted from a pool of $454 \mathrm{HIV}$-infected children that were enrolled into the ART programme of the FMC, Makurdi, between June 2010 and June 2012. The remaining twenty five children whose data were missing for one reason or the other, were excluded from the present study. This cohort of HIV-infected ART naïve children had been previously studied for dyslipidaemia $^{28}$.

Inclusion and exclusion criteria: Included in the study were HIV infected children $(\leq 15$ years of age) who were ART naïve (except for the purpose of prevention of mother-to-child transmission of HIV) and whose venous blood glucose level results were available. HIV-infected ART naïve children with no blood glucose result and/or incomplete records of clinical variables of interest (i.e. demographic, some specific symptoms and signs/physical examination findings, laboratory findings and some comorbidities including tuberculosis, oesophageal candidiasis, diarrhoeal disease, sepsis, malarial fever, pneumonia and undernutrition) and HIVinfected ART-experienced children were excluded.

\section{Operational definitions}

The definition of hypoglycaemia in infants and children continues to be controversial, but for this study, hypoglycaemia was defined as a blood glucose $<2.2 \quad \mathrm{mmol} / 1 \quad(40 \quad \mathrm{mg} / \mathrm{dl})^{29,30}$. Normoglycaemia was blood glucose between $2.2-$ $6.6 \mathrm{mmol} / 1(40-120 \mathrm{mg} / \mathrm{dl})$ and hyperglycaemia was blood glucose over $6.6 \mathrm{mmol} / 1$ (>120 mg/ $\mathrm{dl})^{31,32}$. Abnormal blood glucose, or dysglycaemia, was defined as any of these 2 categories above. Hypocholesterolaemia (total cholesterol value below 160mg/dl) and hypercholestrolaemia (total cholesterol value above $200 \mathrm{mg} / \mathrm{dl}$ ) were according to the Lipid Research Clinics (LRC) prevalence study $^{33}$. To define under-nutrition in children less than 5 years, the weight for height Z-score less than -2 standard deviations ( wasting) from World Health Organization (WHO) reference median was computed using the WHO Anthro software (version $2.0,2008$ ) which was based on WHO child growth standards of $2006^{34}$. For children $\geq 5$ years, the body mass index (BMI) was calculated as the weight in kilograms divided by the square of the height in metres $\left(\mathrm{kg} / \mathrm{m}^{2}\right)$. Values $<18.5 \mathrm{~kg} / \mathrm{m}^{2}$ was defined as under-nutrition, $>25 \mathrm{~kg} / \mathrm{m}^{2}$ as overweight and $>30$ $\mathrm{kg} / \mathrm{m}^{2}$ as obesity ${ }^{35}$. Hepatotoxicity was defined as alanine aminotransferase (ALT) of 1.25-fold over the upper limit of normal of $37 \mathrm{IU} / \mathrm{L}^{36}$. The definitions of "fever", "malaria fever", "diarrhoea", "chronic diarrhoea", "pneumonia", "tuberculosis", "oropharyngeal candidiasis", "oesophageal candidiasis", and "sepsis" were as described in the WHO Pocket Book of Hospital Care for Children: Guidelines for the Management of Common Childhood Illnesses ${ }^{37}$.

The blood glucose was analysed by the glucose oxidase method (GM6 Analox instruments, UK) and it did not require fasting. The total cholesterol was determined enzymatically by cholesterol esterase and cholesterol oxidase and with the Cobas Mira chemistry analyzer. In addition to determining the CD4 count and the viral load, venous blood samples were also collected for haemoglobin concentration, malaria parasites (Giemsa stain), a full blood count (Coulter Micro Diff II, UK), hepatitis B surface antigen and hepatitis $\mathrm{C}$ virus antibody (using the third generation ELISA technique, EIAgen HBsAg Kit, EIAgen $\mathrm{HCV} \mathrm{Ab}$ Kit) and catalytic activity of ALT (determined in serum using a Cobas Mira chemistry analyzer). All tests were done at the APIN/PEPFAR laboratory of FMC, Makurdi. 
Statistical analysis: Descriptive statistics were tabulated as numbers and percentages for categorical variables. The main outcome variable in the analysis was dysglycaemia (i.e. hypoglycaemia and hyperglycaemia considered separately) versus normoglycaemia. The prevalence proportions of hypoglycaemia and hyperglycaemia were calculated. Potential risk factors for dysglycaemia (hypoglycaemia and hyperglycaemia considered separately) were tested for significance in a bivariate logistic regression. These potential risk factors included age and gender, some presenting symptoms and signs on physical examinations and the WHO HIV/AIDS clinical stage, some laboratory findings [CD4 count, alanine aminotransferase (ALT), haemoglobin level, $\mathrm{HBsAg}, \mathrm{HCV}$ antibodies and viral load], and some co-morbidities/opportunistic infections (tuberculosis, oesophageal candidiasis, diarrhoeal disease, sepsis, malarial fever, pneumonia and undernutrition). Risk factors that achieved a significance level of 0.1 at bivariate analyses were modelled into multivariate logistic regression analyses in one block. For all analyses, pvalues less than 0.05 were considered statistically significant. Statistical analysis was done using SPSS version 16.

\section{Results}

There were 206 females and 223 males with a F:M ratio of 1: 1.1. The median age was 5 years with an interquartile range of 3 to 8 years. Twelve children $(2.8 \%, 12 / 429)$ were found with hypoglycaemia and $35(8.2 \%, 35 / 429)$ had hyperglycaemia.

Table1 depicts the risk factors of hypoglycaemia among the cohorts.

Table 1: Risk factors of hypoglycaemia among the cohorts

\begin{tabular}{|c|c|c|c|c|c|c|}
\hline \multirow[t]{2}{*}{ Clinical variable } & \multirow{2}{*}{$\begin{array}{c}\text { Hypoglycaemia } \\
(<2.2 \mathrm{mmol} / \mathrm{l}) \\
\text { Number }(\%)\end{array}$} & \multirow{2}{*}{$\begin{array}{c}\text { Normoglycaemia } \\
(2.2-6.6 \mathrm{mmol} / \mathrm{l}) \\
\text { Number }(\%)\end{array}$} & \multicolumn{3}{|c|}{ Bivariate analysis } & \multirow{2}{*}{$\begin{array}{c}\begin{array}{c}\text { Multivariate } \\
\text { analysis }\end{array} \\
P \text {-value } \\
\end{array}$} \\
\hline & & & COR & $95 \% C I$ & $P$-value & \\
\hline \multicolumn{7}{|c|}{ Demography } \\
\hline $\begin{array}{l}\text { Age (years) } \\
<5 \\
\geq 5 \text { (Ref) }\end{array}$ & $\begin{array}{l}04(33.3) \\
08(66.7)\end{array}$ & $\begin{array}{c}197(47.2) \\
220(52.8)\end{array}$ & 0.558 & $(0.166-1.88)$ & 0.341 & NA \\
\hline $\begin{array}{l}\text { Gender } \\
\text { Male } \\
\text { Female (Ref) }\end{array}$ & $\begin{array}{l}07(58.3) \\
05(41.7)\end{array}$ & $\begin{array}{l}216(51.8) \\
201(48.2)\end{array}$ & 1.30 & $(0.407-4.17)$ & 0.655 & NA \\
\hline \multicolumn{7}{|c|}{ Symptoms } \\
\hline $\begin{array}{l}\text { Irritability } \\
\text { Yes } \\
\text { No (Ref) }\end{array}$ & $\begin{array}{l}10(83.3) \\
02(16.7)\end{array}$ & $\begin{array}{c}04(01.0) \\
413(99.0)\end{array}$ & 516.25 & $(84.53-3153.16)$ & $<0.001$ & 0.999 \\
\hline $\begin{array}{l}\text { Convulsions } \\
\text { Yes } \\
\text { No (Ref) }\end{array}$ & $\begin{array}{l}08(66.7) \\
04(33.3)\end{array}$ & $\begin{array}{c}04(10) \\
413(99.0)\end{array}$ & 185.22 & $(43.74-975.34)$ & $<0.001$ & 0.987 \\
\hline $\begin{array}{l}\text { *Food refusal } \\
\text { Yes } \\
\text { No (Ref) }\end{array}$ & $\begin{array}{c}12(100.0) \\
0(0.0)\end{array}$ & $\begin{array}{r}57(13.7) \\
360(86.3) \\
\end{array}$ & & & & \\
\hline $\begin{array}{l}\text { *Vomiting } \\
\text { Yes } \\
\text { No (Ref) }\end{array}$ & $\begin{array}{c}12(100.0) \\
0(0.0)\end{array}$ & $\begin{array}{c}70(16.8) \\
347(83.2) \\
\end{array}$ & & & & \\
\hline \multicolumn{7}{|c|}{ Physical Signs } \\
\hline $\begin{array}{l}\text { Fever } \\
\text { Yes } \\
\text { No (Ref) }\end{array}$ & $\begin{array}{l}03(25.0) \\
09(75.0)\end{array}$ & $\begin{array}{c}67(16.1) \\
350(83.9)\end{array}$ & 1.74 & $(0.459-6.60)$ & 0.668 & NA \\
\hline $\begin{array}{l}\text { Oral thrush } \\
\text { Yes } \\
\text { No (Ref) }\end{array}$ & $\begin{array}{l}11(91.7) \\
01(08.3)\end{array}$ & $\begin{array}{c}37(08.9) \\
380(91.1)\end{array}$ & 112.97 & $(14.19-899.56)$ & $<0.001$ & 0.786 \\
\hline $\begin{array}{l}\text { Hepatosplenomegaly } \\
\text { Yes } \\
\text { No (Ref) }\end{array}$ & $\begin{array}{l}05(41.7) \\
07(58.3)\end{array}$ & $\begin{array}{c}34(8.2) \\
383(91.8)\end{array}$ & 8.05 & $(2.42-26.75)$ & 0.001 & 0.982 \\
\hline $\begin{array}{l}\text { Hepatomegaly } \\
\text { Yes } \\
\text { No (Ref) }\end{array}$ & $\begin{array}{l}03(25.0) \\
09(75.0)\end{array}$ & $\begin{array}{c}42(10.1) \\
375(89.9)\end{array}$ & 2.98 & $(0.775-11.42)$ & 0.236 & NA \\
\hline $\begin{array}{l}\text { *Splenomegaly } \\
\text { Yes } \\
\text { No (Ref) }\end{array}$ & $\begin{array}{c}0(0.0) \\
12(100.0)\end{array}$ & $\begin{array}{c}05(01.2) \\
412(98.8)\end{array}$ & & & & \\
\hline $\begin{array}{l}\text { WHO clinical staging } \\
1 \& 2 \\
3 \& 4 \text { (Ref) }\end{array}$ & $\begin{array}{c}10(83.3) \\
2(16.7)\end{array}$ & $\begin{array}{c}346(83.0) \\
71(17.0)\end{array}$ & 0.975 & $(0.209-4.54)$ & 0.974 & NA \\
\hline
\end{tabular}

$*=$ Omitted because of collinearity , COR=Crude Odd Ratio, $C I=$ Confidence Interval, $N A=$ Not applicable, $W H O=$ World Health Organization 
Table 1: Risk factors of hypoglycaemia among the cohorts (continued)

\begin{tabular}{|c|c|c|c|c|c|c|}
\hline \multirow[t]{2}{*}{ Clinical variable } & \multirow{2}{*}{$\begin{array}{c}\text { Hypoglycaemia } \\
(<2.2 \mathrm{mmol} / \mathrm{l}) \\
\text { Number }(\%)\end{array}$} & \multirow{2}{*}{$\begin{array}{c}\text { Normoglycaemia } \\
(2.2-6.6 \mathrm{mmol} / \mathrm{l}) \\
\text { Number }(\%)\end{array}$} & \multicolumn{3}{|c|}{ Bivariate analysis } & \multirow{2}{*}{$\begin{array}{c}\begin{array}{c}\text { Multivariate } \\
\text { analysis }\end{array} \\
P \text {-value } \\
\end{array}$} \\
\hline & & & COR & $95 \% C I$ & $P$-value & \\
\hline \multicolumn{7}{|c|}{ Laboratory findings } \\
\hline $\begin{array}{l}\text { CD4 count } \\
\leq 200 \\
>200 \text { ( Ref) }\end{array}$ & $\begin{array}{l}02(16.7) \\
10(83.3)\end{array}$ & $\begin{array}{l}81(19.4) \\
336(80.6)\end{array}$ & 0.830 & $(0.178-3.86)$ & 0.812 & NA \\
\hline $\begin{array}{l}* \text { Viral load (copies } / \mathrm{ml}) \\
<1000 \\
\geq 1000(\text { Ref })\end{array}$ & $\begin{array}{c}0(0.0) \\
12(100.0)\end{array}$ & $\begin{array}{c}38(9.1) \\
379(90.9) \\
\end{array}$ & & & & \\
\hline $\begin{array}{l}\text { Haemoglobin }(\mathrm{g} / \mathrm{dl}) \\
<10 \\
\geq 10 \text { (Ref) }\end{array}$ & $\begin{array}{l}11(91.7) \\
01(08.3)\end{array}$ & $\begin{array}{l}247(59.2) \\
170(40.8)\end{array}$ & 7.57 & $(0.986-59.19)$ & 0.050 & 0.992 \\
\hline $\begin{array}{l}\text { Hepatitis B surface antigen } \\
\text { Yes } \\
\text { No (Ref) }\end{array}$ & $\begin{array}{l}03(25.0) \\
09(75.0)\end{array}$ & $\begin{array}{c}46(11.0) \\
371(89.0)\end{array}$ & 2.69 & $(0.702-10.29)$ & 0.299 & NA \\
\hline $\begin{array}{l}\text { *Hepatitis } \mathrm{C} \text { antibodies } \\
\text { Yes } \\
\text { No (Ref) }\end{array}$ & $\begin{array}{c}0(0.0) \\
12(100.0)\end{array}$ & $\begin{array}{c}11(2.6) \\
406(97.4)\end{array}$ & & & & \\
\hline $\begin{array}{l}\text { Alanine aminotransferase } \\
\text { Elevated }(>46.3) \\
\text { Normal }(\leq 46.3)(\mathrm{Ref})\end{array}$ & $\begin{array}{l}03(25.0) \\
09(75.0)\end{array}$ & $\begin{array}{c}71(17.0) \\
346(83.0)\end{array}$ & 1.62 & $(0.429-6.15)$ & 0.739 & NA \\
\hline $\begin{array}{l}\text { Hypocholesterolaemia } \\
\text { Yes } \\
\text { No (Ref) }\end{array}$ & $\begin{array}{c}11(91.7) \\
01(8.3)\end{array}$ & $\begin{array}{c}368(88.2) \\
49(11.8)\end{array}$ & 1.47 & $(0.185-11.59)$ & 0.716 & NA \\
\hline \multicolumn{7}{|c|}{ Diagnosed co-morbidities /opportunistic infections } \\
\hline $\begin{array}{l}\text { Tuberculosis } \\
\text { Yes } \\
\text { No (Ref) }\end{array}$ & $\begin{array}{l}01(08.3) \\
11(91.7) \\
\end{array}$ & $\begin{array}{c}51(12.2) \\
366(87.8) \\
\end{array}$ & 0.652 & $(0.082-5.16)$ & 0.683 & NA \\
\hline $\begin{array}{l}\text { *Oesophageal candidiasis } \\
\text { Yes } \\
\text { No (Ref) }\end{array}$ & $\begin{array}{c}0(0.0) \\
12(100.0)\end{array}$ & $\begin{array}{c}08(01.9) \\
409(98.1)\end{array}$ & & & & \\
\hline $\begin{array}{l}\text { Diarrhoeal disease } \\
\text { Yes } \\
\text { No (Ref) }\end{array}$ & $\begin{array}{l}01(08.3) \\
11(01.7)\end{array}$ & $\begin{array}{c}11(02.6) \\
406(97.4)\end{array}$ & 3.36 & $(0.398-28.32)$ & 0.238 & NA \\
\hline $\begin{array}{l}\text { *Sepsis } \\
\text { Yes } \\
\text { No (Ref) }\end{array}$ & $\begin{array}{c}0(0.0) \\
12(100.0)\end{array}$ & $\begin{array}{c}14(3.4) \\
403(96.6)\end{array}$ & & & & \\
\hline $\begin{array}{l}\text { Malaria fever } \\
\text { Yes } \\
\text { No (Ref) }\end{array}$ & $\begin{array}{l}03(25.0) \\
09(75.0)\end{array}$ & $\begin{array}{c}54(12.9) \\
363(87.1)\end{array}$ & 2.24 & $(0.588-8.53)$ & 0.435 & NA \\
\hline $\begin{array}{l}\text { Pneumonia } \\
\text { Yes } \\
\text { No (Ref) }\end{array}$ & $\begin{array}{l}04(33.3) \\
08(66.7)\end{array}$ & $\begin{array}{c}21(5.0) \\
396(95.0)\end{array}$ & 9.43 & 0.000 & 0.983 & NA \\
\hline $\begin{array}{l}\text { Weight for height } Z \text { score } \\
<-2 \text { SD } \\
\geq-2 \text { SD (Ref) } \\
\text { Not Counted }=228\end{array}$ & $\begin{array}{l}02(50.0) \\
02(50.0)\end{array}$ & $\begin{array}{c}63(31.9) \\
134(68.1)\end{array}$ & 2.09 & $(0.288-15.22)$ & 0.835 & NA \\
\hline $\begin{array}{l}\text { Body mass index } \\
\geq 18.5 \\
<18.5 \text { (Ref) } \\
\text { Not Counted=201 }\end{array}$ & $\begin{array}{l}07(87.5) \\
01(12.5)\end{array}$ & $\begin{array}{c}186(84.5) \\
34(15.5)\end{array}$ & 1.32 & $(0.158-11.09)$ & 0.796 & NA \\
\hline
\end{tabular}

*=Omitted because of collinearity , COR=Crude Odd Ratio, CI=Confidence Interval, $N A=$ Not applicable,

In bivariate analyses, children with the symptom of convulsions, those with irritability, oral thrush and hepatosplenomegaly on physical examination, and those with co-morbidity of pneumonia were found to be significantly associated with hypoglycaemia. Children with convulsions were associated with a 185 folds risk of hypoglycaemia (OR; 185, 95\%CI; 43.7-975.3, $\mathrm{P}<0.001)$. Also, irritability significantly increased the odds of hypoglycaemia by 516 times (OR; 516.3， 95\%CI; 84.5-3153.2, $\mathrm{P}<0.001$ ). Furthermore, children with oral thrush were 112 times more at risk of having hypoglycaemia (OR; $112.9,95 \% \mathrm{CI} ; 14.2-899.6, \mathrm{P}<0.001)$ and those with hepatosplenomegaly 8 times more (OR; $8.1,95 \% \mathrm{CI}$; 2.4-26.8, $\mathrm{P}=0.001$ ). Pneumonia was also associated with hypoglycaemia (OR; 9.4, 95\%CI; 2.63-33.8, $\mathrm{P}<0.001)$. However at multivariate analyses, none of these risk factors was found to be associated with hypoglycaemia.

Table 2 shows the risk factors of hyperglycaemia among the cohorts. 
Table 2. The risk factors of hyperglycaemia

\begin{tabular}{|c|c|c|c|c|c|c|}
\hline \multirow[t]{2}{*}{ Clinical variables } & \multirow{2}{*}{$\begin{array}{c}\text { Hyperglycaemia } \\
\text { (>6.6mmol/l) } \\
\text { Number }(\%)\end{array}$} & \multirow{2}{*}{$\begin{array}{c}\text { Normoglycaemia } \\
(2.2-6.6 \mathrm{mmol} / \mathrm{l}) \\
\text { Number }(\%)\end{array}$} & \multicolumn{3}{|c|}{ Bivariate analyses } & \multirow{2}{*}{$\begin{array}{c}\begin{array}{c}\text { Multivariate } \\
\text { analyses }\end{array} \\
\text { P-value } \\
\end{array}$} \\
\hline & & & COR & $95 \% C I$ & $P$-value & \\
\hline \multicolumn{7}{|c|}{ Demography } \\
\hline $\begin{array}{l}\text { Age (years) } \\
<5 \\
\geq 5 \text { (Ref) }\end{array}$ & $\begin{array}{l}15(42.9) \\
20(57.1)\end{array}$ & $\begin{array}{l}186(47.2) \\
208(52.8)\end{array}$ & 0.839 & $(0.417-1.69)$ & 0.621 & NA \\
\hline $\begin{array}{l}\text { Gender } \\
\text { Male } \\
\text { Female (Ref) }\end{array}$ & $\begin{array}{l}20(57.1) \\
15(42.9)\end{array}$ & $\begin{array}{l}203(51.5) \\
191(48.5)\end{array}$ & 1.26 & $(0.624-2.52)$ & 0.524 & NA \\
\hline \multicolumn{7}{|l|}{ Symptoms } \\
\hline $\begin{array}{l}\text { *Irritable } \\
\text { Yes } \\
\text { No (Ref) }\end{array}$ & $\begin{array}{c}0(0.0) \\
35(100.0) \\
\end{array}$ & $\begin{array}{c}14(03.6) \\
380(96.4) \\
\end{array}$ & & & & \\
\hline $\begin{array}{l}\text { *Convulsions } \\
\text { Yes } \\
\text { No (Ref) }\end{array}$ & $\begin{array}{c}0(0.0) \\
35(100.0)\end{array}$ & $\begin{array}{c}12(03.0) \\
382(97.0)\end{array}$ & & & & \\
\hline $\begin{array}{l}\text { Food refusal } \\
\text { Yes } \\
\text { No (Ref) }\end{array}$ & $\begin{array}{l}03(08.6) \\
32(91.4)\end{array}$ & $\begin{array}{c}66(16.8) \\
328(83.2)\end{array}$ & 0.466 & $(0.139-1.57)$ & 0.207 & NA \\
\hline $\begin{array}{l}\text { Vomiting } \\
\text { Yes } \\
\text { No (Ref) }\end{array}$ & $\begin{array}{l}04(11.4) \\
31(88.6) \\
\end{array}$ & $\begin{array}{c}78(19.8) \\
316(80.2) \\
\end{array}$ & 0.523 & $(0.179-1.53)$ & 0.228 & NA \\
\hline \multicolumn{7}{|c|}{ Physical Signs } \\
\hline $\begin{array}{l}\text { Fever } \\
\text { Yes } \\
\text { No (Ref) }\end{array}$ & $\begin{array}{l}06(17.1) \\
29(82.9)\end{array}$ & $\begin{array}{c}64(16.2) \\
330(83.8)\end{array}$ & 1.07 & $(0.426-2.67)$ & 0.890 & NA \\
\hline $\begin{array}{l}\text { Oral thrush } \\
\text { Yes } \\
\text { No (Ref) }\end{array}$ & $\begin{array}{l}02(05.7) \\
33(94.3)\end{array}$ & $\begin{array}{c}46(11.7) \\
348(88.3)\end{array}$ & 0.458 & $(0.106-1.97)$ & 0.428 & NA \\
\hline $\begin{array}{l}\text { Hepatosplenomegaly } \\
\text { Yes } \\
\text { No (Ref) }\end{array}$ & $\begin{array}{l}02(05.7) \\
33(94.3) \\
\end{array}$ & $\begin{array}{c}37(09.4) \\
357(90.6)\end{array}$ & 0.585 & $(0.135-2.54)$ & 0.676 & NA \\
\hline $\begin{array}{l}\text { *Hepatomegaly } \\
\text { Yes } \\
\text { No (Ref) }\end{array}$ & $\begin{array}{c}0(0.0) \\
35(100.0)\end{array}$ & $\begin{array}{c}45(11.4) \\
349(88.6)\end{array}$ & & & & \\
\hline $\begin{array}{l}\text { *Splenomegaly } \\
\text { Yes } \\
\text { No (Ref) }\end{array}$ & $\begin{array}{c}0(0.0) \\
35(100.0)\end{array}$ & $\begin{array}{c}05(01.3) \\
389(98.7)\end{array}$ & & & & \\
\hline $\begin{array}{l}\text { WHO Clinical staging } \\
1 \& 2 \\
3 \& 4(\operatorname{Ref})\end{array}$ & $\begin{array}{c}31(88.6) \\
4(11.4)\end{array}$ & $\begin{array}{c}325(82.5) \\
69(17.5)\end{array}$ & 0.608 & $(0.208-1.78)$ & 0.359 & NA \\
\hline
\end{tabular}

*=Omitted because of colinearity, COR=Crude Odds Ratio, CI=Confidence Interval, NA=Not applicable, WHO $=$ World Health Organization 
Table 2. The risk factors of hyperglycaemia (continued)

\begin{tabular}{|c|c|c|c|c|c|c|}
\hline \multirow[t]{2}{*}{ Clinical variables } & \multirow{2}{*}{$\begin{array}{l}\text { Hyperglycaemia } \\
(>6.6 \mathrm{mmol} / \mathrm{l}) \\
\text { Number }(\%)\end{array}$} & \multirow{2}{*}{$\begin{array}{c}\text { Normoglycaemia } \\
(2.2-6.6 \mathrm{mmol} / \mathrm{l}) \\
\text { Number }(\%)\end{array}$} & \multicolumn{3}{|c|}{ Bivariate analyses } & \multirow{2}{*}{$\begin{array}{c}\begin{array}{c}\text { Multivariate } \\
\text { analyses }\end{array} \\
\text { P-value } \\
\end{array}$} \\
\hline & & & COR & $95 \% C I$ & $P$-value & \\
\hline \multicolumn{7}{|c|}{ Laboratory findings } \\
\hline $\begin{array}{l}\text { CD4 count } \\
>200 \text { (Ref) } \\
\leq 200\end{array}$ & $\begin{array}{c}32(91.4) \\
3(8.6)\end{array}$ & $\begin{array}{c}314(79.7) \\
80(20.3)\end{array}$ & 0.368 & $(0.110-1.23)$ & 0.092 & 0.079 \\
\hline $\begin{array}{l}\text { *Viral load (copies/ml) } \\
<1000 \\
\geq 1000 \text { (Ref) }\end{array}$ & $\begin{array}{c}0(0.0) \\
35(100.0)\end{array}$ & $\begin{array}{c}31(07.9) \\
363(92.1)\end{array}$ & & & & \\
\hline $\begin{array}{l}\text { Haemoglobin }(\mathrm{g} / \mathrm{dl}) \\
<10 \\
\geq 10 \text { (Ref) }\end{array}$ & $\begin{array}{l}16(45.7) \\
19(54.3)\end{array}$ & $\begin{array}{l}242(61.4) \\
152(38.6)\end{array}$ & 0.529 & $(0.264-1.06)$ & 0.069 & 0.059 \\
\hline $\begin{array}{l}\text { Hepatitis B surface antigen } \\
\text { Yes } \\
\text { No (Ref) }\end{array}$ & $\begin{array}{l}04(11.4) \\
31(88.6)\end{array}$ & $\begin{array}{c}45(11.4) \\
349(88.6)\end{array}$ & 1.00 & $(0.338-2.97)$ & 0.999 & NA \\
\hline $\begin{array}{l}\text { Hepatitis } \mathrm{C} \text { antibodies } \\
\text { Yes } \\
\text { No (Ref) }\end{array}$ & $\begin{array}{l}03(08.6) \\
32(91.4)\end{array}$ & $\begin{array}{c}08(02.0) \\
386(98.0)\end{array}$ & 4.51 & $(1.14-17.85)$ & 0.019 & $0.029 \dagger$ \\
\hline $\begin{array}{l}\text { Alanine aminotransferase } \\
\text { Elevated }(>46.3) \\
\text { Normal }(\leq 46.3) \text { (Ref) }\end{array}$ & $\begin{array}{l}06(17.1) \\
29(82.9) \\
\end{array}$ & $\begin{array}{c}68(17.3) \\
326(82.7) \\
\end{array}$ & 0.992 & $(0.396-2.482)$ & 0.986 & NA \\
\hline $\begin{array}{l}\text { Hypocholestrolemia } \\
\text { Yes } \\
\text { No (Ref) }\end{array}$ & $\begin{array}{l}31(88.6) \\
04(11.4)\end{array}$ & $\begin{array}{c}348(88.3) \\
46(11.7)\end{array}$ & 1.02 & $(0.346-3.03)$ & 0.965 & NA \\
\hline \multicolumn{7}{|c|}{ Diagnosed co-morbidities / opportunistic infections } \\
\hline $\begin{array}{l}\text { Tuberculosis } \\
\text { Yes } \\
\text { No (Ref) }\end{array}$ & $\begin{array}{l}02(05.7) \\
33(94.3)\end{array}$ & $\begin{array}{c}50(12.7) \\
344(87.3)\end{array}$ & 0.417 & $(0.097-1.79)$ & 0.346 & NA \\
\hline $\begin{array}{l}\text { Esophageal candidiasis } \\
\text { Yes } \\
\text { No (Ref) }\end{array}$ & $\begin{array}{l}02(05.7) \\
33(94.3) \\
\end{array}$ & $\begin{array}{c}06(01.5) \\
388(98.5) \\
\end{array}$ & 3.92 & $(0.761-20.19)$ & 0.269 & NA \\
\hline $\begin{array}{l}\text { *Diarrhoeal disease } \\
\text { Yes } \\
\text { No (Ref) }\end{array}$ & $\begin{array}{c}0(0.0) \\
35(100.0)\end{array}$ & $\begin{array}{c}12(03.0) \\
382(97.0)\end{array}$ & & & & \\
\hline $\begin{array}{l}\text { Sepsis } \\
\text { Yes } \\
\text { No (Ref) }\end{array}$ & $\begin{array}{l}01(02.9) \\
34(97.1)\end{array}$ & $\begin{array}{c}13(03.3) \\
381(96.7)\end{array}$ & 0.862 & $(0.109-6.79)$ & 0.888 & NA \\
\hline $\begin{array}{l}\text { Malaria fever } \\
\text { Yes } \\
\text { No (Ref) }\end{array}$ & $\begin{array}{l}08(22.9) \\
27(77.1)\end{array}$ & $\begin{array}{c}49(12.4) \\
345(87.6) \\
\end{array}$ & 2.09 & $(0.897-4.85)$ & 0.139 & NA \\
\hline $\begin{array}{l}\text { Pneumonia } \\
\text { Yes } \\
\text { No (Ref) }\end{array}$ & $\begin{array}{l}01(02.9) \\
34(97.1)\end{array}$ & $\begin{array}{c}24(06.1) \\
370(93.9)\end{array}$ & 0.453 & $(0.059-3.45)$ & 0.685 & NA \\
\hline $\begin{array}{l}\text { Weight for Height } Z \text { score } \\
<-2 \text { SD } \\
\geq-2 \text { SD (Ref) } \\
\text { Not counted }=228\end{array}$ & $\begin{array}{l}03(20.0) \\
12(80.0)\end{array}$ & $\begin{array}{c}62(33.3) \\
124(66.7)\end{array}$ & 0.492 & $(0.134-1.80)$ & 0.423 & NA \\
\hline $\begin{array}{l}\text { Body Mass Index } \\
\geq 18.5 \\
<18.5 \text { (Ref) } \\
\text { Not counted = } 201\end{array}$ & $\begin{array}{l}14(70.0) \\
06(30.0)\end{array}$ & $\begin{array}{c}179(86.1) \\
29(13.9)\end{array}$ & 3.91 & $(0.139-1.10)$ & 0.131 & NA \\
\hline
\end{tabular}

*=Omitted because of colinearity, COR=Crude Odds Ratio, CI=Confidence Interval, NA=Not applicable, $W H O=$ World Health Organization. Adjusted Odds Ratio 2.06, 95\% CI; 1.05-8.52

In both bivariate and multivariate analyses, only Hepatitis C co-infection was found to be significantly associated with the risk of hyperglycaemia. At the adjusted regression model, the trend was such that subjects who were coinfected with Hepatitis $\mathrm{C}$ were at significant risk of hyperglycaemia compared to those who had mono infection with HIV (Adjusted Odds Ratio 2.06, 95\% CI; $1.05-8.52, \mathrm{P}=0.03)$.

\section{Discussion}

This is the first study that described the burden and the risk factors of dysglycaemia among Nigerian HIV-infected ART-naïve children. The study reveals that whilst the prevalence of hypoglycaemia was low at $2.8 \%$, that of hyperglycaemia was high at $8.2 \%$. The prevalence of hypoglycaemia of $2.8 \%$ in this study was lower than the $5.6 \%$ and $6.4 \%$ in a cohort of Nigerian children admitted at the emergency paediatric units in Lagos ${ }^{38}$ and Ile-Ife ${ }^{39}$ 
respectively. In other settings outside Nigeria, the prevalence of hypoglycaemia of $2.8 \%$ in this study was lower than the respective $7.1 \%$ and $8.2 \%$ among paediatric admissions in Mozambique ${ }^{40}$ and in Kenya $^{30}$. Apart from Lagos and Ile-Ife studies which used a higher cut-off value of less than 2.5 $\mathrm{mmol} / 1$ to define hypoglycaemia, the Kenyan ${ }^{30}$ and Mozambique $^{40}$ studies utilized the same value of less than $2.2 \mathrm{mmol} / \mathrm{l}$ with ours, thereby underscoring the fact that the prevalence of hypoglycaemia was in fact higher in their own cohorts ${ }^{30,40}$. In making comparisons however, caution must be exercised because of differences in the composition of the subjects that populated the different studies ${ }^{30,38-40}$. Ours was essentially among HIV-infected ARTnaïve children.

It is also noteworthy that apart from pneumonia which was found to be significantly associated with hypoglycaemia in bivariate analyses, some other known confounders of hypoglycaemia including malarial infestations, sepsis, undernutrition and diarrhoeal disease ${ }^{18-24}$ were not seen to be associated with hypoglycaemia in this study. This finding is in contrast to that of Ile-Ife ${ }^{39}$ where severa malaria, sepsis, pneumonia and malnutrition were found to be associated with hypoglycaemia and to that of Lagos $^{38}$ where severe malaria, sepsis, marasmus and diarrhoeal disease were found to be associated with hypoglycaemia. In Mozambique ${ }^{40}$ hypoglycaemia was found to be significantly associated with severe malaria, protein energy malnutrition, and pneumonia. In $\mathrm{Kenya}^{30}$, malaria and malnutrition were also associated with hypoglycaemia.

The pathophysiology of hypoglycaemia in pneumonia may be due to a combination of mechanisms including increased glucose utilization by macrophage-rich tissues of the liver, lung, spleen, ileum, and skin and also from depressed hepatic gluconeogenesis that may result from the decreased sensitivity to stress hormones and/or adrenal failure, both of which can be triggered by pulmonary infections ${ }^{29}$. There was also a significant association at bivariate analyses between convulsions, irritability, oral thrush, hepatomegaly and hypoglycaemia.

Irritability and convulsions are known neurological symptoms of hypoglycaemia and as such, the significant association between these symptoms and hypoglycaemia may not be too difficult to explain. However, the absence of these clinical symptoms does not always indicate that the glucose concentration is normal or that it has not fallen below optimal level for brain metabolism ${ }^{38}$. Although subjects with oral thrush may be expected to eat poorly, this alone cannot explain the association with hypoglycaemia as "food refusal", surprisingly, was not found to be associated with hypoglycaemia in these subjects. The interaction with other confounders in the multivaraiate analysis may have reduced the importance of oral thrush as a risk factor of hypoglycaemia in this study. Hepatosplenomegaly depicts a progression in HIV disease and may also accompany many infectious diseases that may be seen in HIV/AIDS and as such, hepatosplenomegaly may be associated with hypoglycaemia of infectious diseases as earlier explained $^{12,29}$.

Among paediatric admissions into hospitals, the prevalence of hyperglycaemia of $8.2 \%$ in the present study was lower than $10.9 \%$ reported by Sambany et al in Magadascar ${ }^{29}$, the $25 \%$ by Palacio et al in Atlanta, $\mathrm{USA}^{31}$, but was higher than the $2.7 \%$ reported by Osier et al in Kenya ${ }^{30}$. The various definitions ascribed to hyperglycaemia could explain the different prevalence of hyperglycaemia in our study and those of others ${ }^{29-31}$. Whilst we used $>6.6 \mathrm{mmol} / \mathrm{l}$ as a cut-off value for hyperglycaemia in our own study, it was $>8.3 \mathrm{mmol} / 1,>7.1 \mathrm{mmol} / 1$ and $>10 \mathrm{mmol} / 1$ in the studies in Madagascar, Atlanta and Kenya respectively. Also, the different spectrum of diseases among our HIV-infected ARTnaive children and the children in other settings ${ }^{29-31}$ could also explain the varying burden of hyperglycaemia.

Although there is a dearth of data on the prevalence of hyperglycaemia among HIV-infected ART naive children, the prevalence of $8.2 \%$ in our study and $21.5 \%$ in the study of Shen et al ${ }^{41}$ among the adult population in China, have shown that the prevalence of hyperglycaemia in untreated HIV-infection persons could be high. Dube ${ }^{11}$ had earlier suggested that there may be an insulin resistance occurring as an association of HIV-disease and thus may provide an explanation for the hyperglycaemia of HIVinfection. Also, the stress of HIV-infection can cause elevations in plasma concentrations of hyperglycaemic hormones such as glucocorticoids, catecholamines, glucagon and growth hormones with a resultant alterations in the metabolism of glucose and other energy substrates and may thus explain the hyperglycaemia seen ${ }^{29}$.

In this study, only hepatitis $\mathrm{C}$ was independently associated with hyperglycaemia in multivariate analyses. Wohl et $\mathrm{al}^{42}$ had also mentioned hepatitis $\mathrm{C}$ infection as a risk factor for hyperglycaemia among HIV-infected adults. The pathophysiology of hyperglycaemia in hepatitis $\mathrm{C}$ infection is not completely understood. However, studies have suggested that an expression of the hepatitis $\mathrm{C}$ virus core protein can induce hepatic insulin resistance through alterations in insulin receptor substrate-1 pathway. With a progressive increase in insulin resistance, the compensatory increase in insulin 
secretion by the $\beta$-cell reaches fails and hyperglycaemia thus emerges ${ }^{43}$.

There are some limitations to the study. It was not determined if the hyperglycaemia at the point of recruitment represents a diagnosis of diabetes mellitus or just a response to the stress of HIV infection in our cohort. Unfortunately, being a retrospective study, a longitudinal follow-up of serum glucose level was not done. Also, the range of the potential risk factors that were evaluated for dysglycaemia was limited by the retrospective design of the study. Furthermore, the prevalence of dysglycaemia may have been underestimated as many more critically ill HIV-infected ART naïve children presented at the Emergency Unit of the FMC, Makurdi, rather than at the ART clinic where the data was abstracted.

\section{Conclusions}

- In this study HIV-infected Nigerian children who were not on ART had a low prevalence of hypoglycaemia but a high prevalence of hyperglycaemia.

- Hepatitis C co-infection was a significant independent risk factor of hyperglycaemia.

\section{References}

1. UNICEF. State of the World Children, 2015. Available from: http://www.data.unicef.org/corecode/uplo ads/document6/uploaded_pdfs/corecode/S OWC_2015_all-countriesupdate_214.xlsx.

2. Obimbo EM, Mbori-Ngacha DA, Ochieng JO, Richardson BA, Otieno PA, Bosire R, et al. Predictors of early mortality in a cohort of human immunodeficiency virus type 1-infected African children. Pediatric Infectious Disease Journal 2004; 23:53643.

https://doi.org/10.1097/01.inf.0000129692 .42964 .30

PMid: 15194835 PMCid: PMC3380074

3. Gibb DM, Duong T, Tookey PA, Sharland M, Tudor-Williams G, Novelli V, et al. National Study of HIV in Pregnancy and Childhood Collaborative HIV Paediatric Study. Decline in mortality, AIDS, and hospital admissions in perinatally HIV-1 infected children in the United Kingdom and Ireland. British Medical Journal 2003; 327:1019.

https://doi.org/10.1136/bmj.327.7422.101

9

PMid: 14593035 PMCid: PMC261655
4. Sauvageot D, Schaefer M, Olson D, Pujades-Rodriguez M, O'Brien DP. Antiretroviral therapy outcomes in resource-limited settings for $\mathrm{HIV}$-infected children $<5$ years of age. Pediatrics 2010; 125: 1039-47.

https://doi.org/10.1542/peds.2009-1062

PMid: 20385636

5. Sinha U, Sengupta N, Mukhopadhyay P, Roy KS. Human immunodeficiency virus endocrinopathy. Indian Journal of Endocrinology and Metabolism 2011; 15(Suppl 4): 251-60. https://doi.org/10.4103/2230-8210.85574

PMid: 22028995 PMCid: PMC3193773

6. Unachukwu CN, Uchenna DI, Young EE. Endocrine and metabolic disorders associated with human immune deficiency virus infection. West African Journal of Medicine 2009 ; 28(Supp11):3-9.

https://doi.org/10.4314/wajm.v28i1.48415

PMid: 19662737

7. Sellmeyer DE, Grunfeld C. Endocrine and metabolic disturbances in human immunodeficiency virus infection and the acquired immune deficiency syndrome. Endocrine Reviews 1996; 17:518-32. https://doi.org/10.1210/edrv-17-5-518

8. Perronne C, Bricaire F, Leport C, Assan D, Vilde J L, Assan R. Hypoglycaemia and diabetes mellitus following parenteral pentamidine mesylate treatment in AIDS patients. Diabetic Medicine 1990; 7: 585-9. https://doi.org/10.1111/j.14645491.1990.t b01453.x

PMid: 2146064

9. Carr A, Samaras K, Thorisdottir A, Kaufmann GR, Chisholm DJ, Cooper DA. Diagnosis, prediction, and natural course of HIV-1 protease inhibitor-associated lipodystrophy, hyperlidaemia and diabetes mellitus: a cohort study. Lancet 1999; 335:2093-9.

https://doi.org/10.1016/S01406736(98)08 468-2

10. Martinez E, Conget I, Lozano L, Casamitjana R, Gatell JM. Reversion of metabolic abnormalities after switching from HIV-1 protease inhibitors to nevirapine. AIDS 1999; 13:805-10. 
https://doi.org/10.1097/000020301999050

70-00009

PMid: 10357379

11. Dube MP. Disorders of glucose metabolism in patients infected with human immunodeficiency virus. Clinical Infectious Diseases 2000; 31:1467-75. https://doi.org/10.1086/317491

PMid: 11096014

12. Hommes MJT, Romihn JA, Endert E, Schattenkerk JKME, Sauerwein HP. Insulin sensitivity and insulin clearance in human immunodeficiencv virus infectedmen. Metabolism 1991; 40:651-6. https://doi.org/10.1016/00260495(91)9005 9-6

13. Aurpibul L, Puthanakit T, Lee B, Mangklabruks A, Sirisanthana T, Sirisanthana V. Lipodystrophy and metabolic changes in HIV-infected children on non-nucleoside reverse transcriptase inhibitor-based antiretroviral therapy. Antiviral Therapy 2007; 12:124754.

PMid: 18240864

14. Fetuga MB, Ogunfowora OB, Oyegunle $\mathrm{VN}$, Thanni LOA. A ten-year review of paediatric HIV/AIDS among hospitalized children in a Nigerian Teaching hospital. Nigerian Journal of Paediatrics 2005; 32(Suppl 3): 29-32.

15. Temiye EO, Akinsulie AO, Ezeaka CV, Adetifa IMO,Iroha EO, Grange AO. Constraints and prospects in the management of Pediatric HIV/AIDS. Journal of the National Medical Association 2006; 98(Suppl 8):1252-9. PMid: 16916121 PMCid: PMC2569553

16. Bugaje MA, Aikhionbare HA. Paediatrics HIV/AIDS seen at Ahmadu Bello University Teaching Hospital, Zaria, Nigeria. Annals of African Medicine 2006; 5(Suppl 2): 73-7.

17. Ojukwu JU, Ogbu CN. Paediatrics HIV/AIDS in Abakaliki. Nigerian Journal of Paediatrics 2003; 30(Suppl 4):128-34.

18. Don M, Valerio G, Korppi M, Canciani M. Hyper- and hypoglycaemia in children with community-acquired pneumonia. Journal of Pediatric Endocrinology and Metabolism 2008; 21(Supp1 7):657-64. https://doi.org/10.1515/JPEM.2008.21.7.6 57

PMid: 18780600

19. World Health Organisation. Management of the child with a serious infection or severe malnutrition. Guidelines at the first referral level in developing countries. Geneva: World Health Organisation, 2000.

20. White NJ, Miller KD, Marsh K, et al. Hypoglycaemia in African children with severe malaria. Lancet 1987; 1: 708-11. https://doi.org/10.1515/JPEM.2008.21.7.6 57

PMid: 18780600

21. Jan IS, Tsai TH, Chen JM, Jerng JS, Hsu $\mathrm{HF}$, et al. Hypoglycemia associated with bacteremic pneumococcal infections. International Journal of Infectious Diseases 2009; 13: 570-6. https://doi.org/10.1016/j.ijid.2008.08.026 PMid: 19071049

22. Bennish ML, Azad AK, Rahman O, et al. Hypoglycemia during diarrhea in childhood. Prevalence, pathophysiology, and outcome. New England Journal of Medicine 1990; 322:1357-63. https://doi.org/10.1056/NEJM1990051032 21905

PMid: 2325734

23. Ronan A, Azad AK, Rahman O, et al. Hyperglycaemia during childhood diarrhoea. Journal of Paediatrics 1997; 130:45-51.

https://doi.org/10.1016/S00223476(97)70 309-3

24. Branco RG, Garcia PC, Piva JP, Casartelli $\mathrm{CH}$, Seibel V, Tasker RC: Glucose level and risk of mortality in pediatric septic shock. Pediatric Critical Care Medicine 2005; 6:470-2.

https://doi.org/10.1097/01.PCC.00001612 84.96739.3A

25. Hirshberg E, Larsen G, Van Duker H. Alterations in glucose homeostasis in the paediatric intensive care unit: Hyperglycaemia and glucose variability are associated with increased mortality and 
morbidity. Pediatric Critical Care Medicine 2008; 9 (Suppl 4): 361-6. https://doi.org/10.1097/PCC.0b013e31817 2d401

PMid:18496414

26. Wintergerst KA, Buckingham B, Gandrud L, Wong BJ, Kache S, Wilson DM. Association of hypoglycaemia, hyperglycaemia and glucose variability with morbidity and death in the pediatric intensive care unit. Pediatrics 2006 ;118 (Suppl 1):173-9.

https://doi.org/10.1542/peds.2005-1819

PMid:16818563

27. Kish L. Survey sample. John Wiley and Sons, New York; 1965.

28. Anigilaje EA, Olutola A. High prevalence of hypocholesterolaemia among antiretroviral therapy-naïve HIV-infected children in Makurdi, Nigeria: A retrospective study. International Journal of Tropical Disease and Health 2014; 4(Suppl 11): 1132-45.

https://doi.org/10.9734/IJTDH/2014/1152 3

29. Sambany E, Pussard E, Rajaonarivo C, Raobijaona H, Barennes H. Childhood dysglycaemia: Prevalence and outcome in a referral hospital. PLOS ONE 2013; 8(5): e65193.

https://doi.org/10.1371/journal.pone.0065

193

PMid: 23741481 PMCid: PMC3669285

30. Osier FHA, Berkley JA, Ross A, Sanderson F, Mohammed S, Newton CRJC. Abnormal blood glucose concentrations on admission to a rural Kenyan district hospital: prevalence and outcome. Archives of Disease in Childhood 2003; 88:621-5. https://doi.org/10.1136/adc.88.7.621 PMid: 12818911 PMCid: PMC1763181

31. Palacio A, Ceron M, Klein R, Cho IS, Smiley D, Mejia R, et al. Prevalence and clinical outcome of inpatient hyperglycaemia in a community paediatric hospital. Journal of Hospital Medicine 2008; 3(Suppl 3): 212-7.

https://doi.org/10.1002/jhm.309

PMid: 18570331 PMCid: PMC3711122
32. Faustino EV, Apkon M. Persistent hyperglycaemia in critically ill children. Journal of Pediatrics 2005; 146:30-4. https://doi.org/10.1016/j.jpeds.2004.08.07 6

PMid: 15644818

33. The Lipid Research Clinics Population Studies Data Book. Vol 1. Bethesda,MD:National Institutes of Health; Lipid Metabolism Branch; Division of Heart and Vascular Diseases; National Heart, Lung, and Blood Institute; 1980.

34. World Health Organization (WHO) Anthro for personal computers, version 3.2.2, 2011.Software for assessing the growth and development of the world's children, Child growth standards. 2011. http://www.who.int/childgrowth/software/ anthro_pc_manual_v322.pdf. Accessed Dec 2012.

35. World Health Organization. (1995) Physical status: the use and interpretation of anthropometry. Report of a WHO Expert Committee. WHO Technical Report Series 854. Geneva: World Health Organization. http://whqlibdoc.who.int/trs/WHO_TRS_ 854.pdf?ua=1. Accessed Feb $201 \overline{4}$.

36. HIV/AIDS, Antiretroviral therapy for HIV Infection in infants and children: Towards universal access. Recommendations for a public health approach: 2010 Revision, 2010.

37. WHO. Pocket Book of Hospital Care for Children: Guidelines for the Management of Common Childhood Illnesses. 2nd ed. WHO: Geneva; 2013. Available from: http://apps.who.int/iris/bitstream/10665/81 170/1/9789241548373_eng.pdf. Accessed February 11, 2015.

38. Oyenusi EE,Oduwole AO, Oladipo OO, Njokanma OF, Esezobor CI. Hypoglycaemia in children aged 1 month to 10 years admitted to the children's emergency centre of Lagos University Teaching Hospital, Nigeria.

South African Journal of Child Health 2014; 8(Suppl 3):107-11. https://doi.org/10.7196/sajch.737 
39. Elusiyan JB, Adejuyigbe EA, Adeodu OO. Hypoglycaemia in a Nigerian paediatric emergency ward. Journal of Tropical Pediatrics 2006;52(Suppl 2):96-102. https://doi.org/10.1093/tropej/fmi068

PMid: 16169861

40. Solomon T, Felix JM, Samuel M, et al. Hypoglycaemia in paediatric admissions in Mozambique. Lancet 1994; 343:149-50. https://doi.org/10.1016/S01406736(94)90 937-7

41. Shen Y, Wang Z, Liu L, Zhang R, Zheng $\mathrm{Y}, \mathrm{Lu}$ H.Prevalence of hyperglycaemia among adults with newly diagnosed HIV/AIDS in China. BMC Infectious Diseases 2013; 13:79.

https://doi.org/10.1186/1471-2334-13-79

PMid: 23394285 PMCid: PMC3574853
42. Wohl DA, McComsey G, Tebas P, Brown TT, Glesby MJ, Reeds D, et al. Current concepts in the diagnosis and management of metabolic complications of HIV infection and Its therapy. Clinical Infectious Diseases 2006; 43:645-53. https://doi.org/10.1086/507333

PMid: 16886161

43. Naing C, Mak JW, Ahmed SI, Maung M. Relationship between hepatitis $\mathrm{C}$ virus infection and type 2 diabetes mellitus: Meta-analysis. World Journal of Gastroenterology 2012; 18 (Suppl 14): 1642-51. https://doi.org/10.3748/wjg.v18.i14.1642 PMid: 22529694 PMCid: PMC3325531 